\title{
Changes in Microcirculation as Early Markers for Infection in Preterm Infants-An Observational Prospective Study
}

\author{
KATHRIN WEIDLICH, JULIA KROTH, CLAUDIA NUSSBAUM, STEPHAN HIEDL, ANDREAS BAUER, FRANK CHRIST, \\ AND ORSOLYA GENZEL-BOROVICZENY
}

\author{
Department of Internal Medicine [K.W.], General Hospital Munich, Munich Bavaria 80804, Germany; Clinic of Dermatology and \\ Allergology [J.K.], University of Munich; Department of Gynecology and Obstetrics [C.N., S.H., O.G.-B.], University Children's Hospital \\ University of Munich, Munich Bavaria 80337, Germany; Clinic for Anesthesiology [A.B., F.C.], University of Munich, Grosshadern, \\ Munich Bavaria 81366, Germany
}

\begin{abstract}
In adults with severe sepsis, the disturbances of the sublingual microcirculation can be quantified with orthogonal polarization spectral imaging. We investigated the cutaneous microcirculation of preterm infants with proven infection (PosInf) and with suspected but unproven infection (NegInf). In 25 infants, orthogonal polarization spectral images were obtained daily, videos of the images were blinded, and analyzed off-line. Functional small vessel density (FSVD) was prospectively calculated from day 3 to day 30 of life. There were 17 episodes of proven and nine episodes of suspected but unproven nosocomial late onset infection. Four infants remained healthy. The data were analyzed for the $5 \mathrm{~d}$ before the start of antibiotics (day -5 until day -1 ). FSVD varied widely, but in the PosInf-group, we found a $10 \%$ decline from day -5 to day -1 ( $p=$ $0.013)$. There was no significant change over time in the NegInfgroup ( $p=0.58$ ). Thus, in infants with proven infection, FSVD decreases already $1 \mathrm{~d}$ before changes in laboratory parameters. However, these changes in FSVD during infection are not represented by absolute values, but must be identified by daily intraindividual observation. (Pediatr Res 66: 461-465, 2009)
\end{abstract}

$\mathrm{V}$ ery low-birth weight infants are at increased risk for episodes of nosocomial infection, which contributes significantly to mortality and morbidity (1). Early diagnosis and prompt administration of appropriate antibiotics are crucial to improve outcome but clinical signs of infection are very unspecific. White blood cell count has not been shown to improve early diagnosis; C-reactive protein (CRP) is quite specific but not very sensitive for neonatal infection. Cytokines such as interleukine (IL)-6 increase early in neonatal infection before the rise of CRP, and the combination of both increases sensitivity and specificity (2). The need for early treatment in combination with nonspecific clinical signs leads to significant iatrogenic blood loss (3) and an increased exposure to antibiotics.

Most clinical signs of infection such as change of skin color, a prolonged capillary filling time, and temperature instability are caused by altered microcirculation. Changes in microcirculation play an important role in the development of septic organ dysfunction $(4,5)$ and the severity of change may even predict outcome (6). Improved technology has made

Received January 5, 2009; accepted June 9, 2009

Correspondence: Orsolya Genzel-Boroviczény, M.D., Department of Gynecology and Obstetrics, University Children's Hospital University of Munich, Maistr. 11, 80337 Munich, Germany; e-mail: genzel@med.uni-muenchen.de observation and quantification of microcirculatory parameters possible. One of the most promising instrumentation in the regard is the orthogonal polarization spectral (OPS) imaging technique, which allows new insights in the human microcirculation and semiquantified assessment. This method provides high-resolution images of the microvascular architecture to a depth of $1 \mathrm{~mm}$. OPS has been validated by multiple studies in animals and humans $(7,8)$. Using OPS Sakr et al. (6) recently found an association between microcirculatory alterations, organ dysfunction, and death in patients with septic shock. We have previously shown that OPS imaging and measurement of small vessel density can be obtained of the skin of premature infants $(9,10)$.

The objective of this study was to prospectively investigate the microcirculation in preterm infants with OPS at the onset of infection.

\section{METHODS}

Patients. For a period of $6 \mathrm{mo}$, all preterm infants with a gestational age $<30$ wk admitted to the intensive care unit were enrolled in a larger microcirculation study between days 1 and 3 of life after parental consent. Details of this study have been previously reported (10). Gestational age in completed weeks was determined by maternal dates of the last menstrual period and by early ultrasound measurements and was confirmed by clinical examination. Infants with congenital malformations were excluded. The institutional review board of the Medical Faculty of the Ludwig-MaximiliansUniversity Munich approved the study protocol and the consent forms.

We obtained daily video sequences of $10 \mathrm{~s}$ duration each from five different spots of the upper inner arm of each patient for the first $30 \mathrm{~d}$ of life. Heart rate, systolic, diastolic, and mean arterial blood pressures were recorded simultaneously with the Dinamap (Critikon Vital Monitor, Norderstedt, Germany). Body core temperature with a skin probe over the liver was also recorded, as were incubator temperature and skin temperature at the OPS measurement point. $\mathrm{Hb}$ or hematocrit levels were obtained from the charts. For a standardized clinical approach to suspected infection, medical and nursing staff separately completed daily a questionnaire assessing the infant in regard to possible signs of infection. The questionnaire was summarized to an overall score. The physicians' questionnaire contained six items of their daily physical examination (paleness; palpable liver size, abdominal examination; cool extremities; irritability; and capillary filling time) and the nurses' questionnaire contained five items (paleness, general clinical impression, abdominal girth, irritability, and feeding intolerance).

Abbreviations: CRP, C-reactive protein; FSVD, functional small vessel density; NegInf, biochemically unproven infection; OPS, orthogonal polarization spectral; PosInf, biochemically proven infection; RBC Vel, red blood cell velocity 
If infection was suspected by the staff, CRP, IL-6 levels, blood, and urine cultures were obtained as part of standard of care. If all cultures and laboratory values remained negative (CRP $<0.5 \mathrm{mg} / \mathrm{dL}$ and IL- $6<10 \mathrm{pg}$ / $\mathrm{mL}$ ), antibiotics were stopped after 48 to $72 \mathrm{~h}$. Infants with proven infection were treated until CRP values fell below $<0.5 \mathrm{mg} / \mathrm{dL}(11,12)$. Treatment decisions were not influenced by the study, and microcirculatory parameters were not known until after the end of the study.

OPS imaging. OPS imaging consists of a cold green light source that is shining through the tissue and with the use of special optics and after multiple scattering events, a virtual light source is created within the tissue at a depth of $\sim 1 \mathrm{~mm}$. The light shining back through the tissue is absorbed by $\mathrm{Hb}$, yielding an image of the illuminated vessel in negative contrast with a resolution of 1 pixel $\approx 1 \mu \mathrm{m}$. The method has been validated for quantitative measurements of microcirculatory parameters in animal model against intravital fluorescence microscopy (7). As in our previous studies, images were obtained with the use of CYTOSCAN A/R from the inner upper arm near the axilla with the least possible contact to avoid pressure against the skin $(9,10)$. As previously described, a small drop of normal saline warmed to incubator temperature was used for better contact $(9,10)$. Skin temperature did not change when applied. Sedation was not necessary.

Images were stored on videotape and analyzed off-line. Functional small vessel density (FSVD), red blood cell velocity (RBC Vel) and vessel diameter were calculated with the use of CapiScope program in as many vessels as possible per observation area. To measure the diameter, a vessel is selected from the image on the video screen and a line is placed in the middle of the vessel. The program calculates the diameter from 1 pixel perpendicular lines placed over the whole length of the drawn line, based on gray level change. RBC Vel is measured using a spatial correlation technique, which requires a 10 -s video sequence in which the tissue reveals little movement. Vessel density is defined as the length of RBC-perfused vessels per observation unit area and is given as centimeter per square centimeter. For this article, the expression "vessel density" refers to perfused vessels (FSVD). In neonates, because of the small size of vessel, arterioles, capillaries, and postcapillary venules cannot be clearly distinguished, and so calculations were done in vessels of diameter ranged from 6 to $24 \mu \mathrm{m}$ (CapiScope: www.kktechnology. com/help/capiscope.html).

Because heterogeneity of flow seems to be an important characteristic of microvascular alterations during sepsis, we analyzed the sequences from day -5 to day 0 with a scoring system adapted from the method of De Backer and Boerma (13-15). Briefly, three equidistant horizontal and three vertical lines were drawn. The type of flow from vessel cutting one of the lines was defined as continuous or pathologic (intermittent, sluggish, or absent). Values are given as percent of pathologic flow of the total number of vessels crossing a vertical horizontal line. Only vessels with diameter $<24 \mu \mathrm{m}$ were included.

The images were allocated random numbers, and all images from all infants during the entire study time were analyzed by two of the investigators (K.W. and J.K.), who were, therefore, blinded to the clinical status of the infant at the time of the analysis. Only after all images were analyzed, data were grouped according to the infants' clinical status. The two investigators analyzed the images of the first 10 infants together until variation of FSVD was $<2 \%$.

The infants were retrospectively divided in 3 groups: PosInf group, infants with proven infection by laboratory parameters and/or positive blood culture; NegInf group, infants with suspected but not proven infection; and healthy group, infants who never had any episode of suspected or proven infection and did not receive any antibiotic treatment during the first $30 \mathrm{~d}$ of life. We defined the day when antibiotic treatment was started as day 0 of infection, and evaluation was concentrated on $5 \mathrm{~d}$ before (day -5 until day -1 ) and $5 \mathrm{~d}$ after (day 1 until day 5) day 0.

Statistics. Data are presented as median with $95 \%$ confidence interval (CI). Data were analyzed using Mann-Whitney $U$ test for unpaired and the Wilcoxon test for paired data. For correlation, the Spearman rank two-way test was applied. The level of significance was set at $p<0.05$, by repeated tests at $p<0.01$.

All calculations were done with GraphPad Prism 3.0 for Windows 95 (GraphPad Software, Inc., San Diego, CA).

\section{RESULTS}

Clinical data. All 25 preterm infants born during the 6-mo study period were enrolled in the study. Gestational age ranged from 23 to 29 wk and birth weight from 570 to $1488 \mathrm{~g}$ (Table 1). All 25 infants survived the first $30 \mathrm{~d}$ of life. We observed 17 episodes of proven infection in 14 preterm infants (PosInf group). There were nine episodes of
Table 1. Clinical data of the 21 preterm infants as median (95\% CI)

\begin{tabular}{lcc}
\hline & $\begin{array}{c}\text { PosInf group } \\
14 \text { preterm } \\
\text { infants 17 proven } \\
\text { infections }\end{array}$ & $\begin{array}{c}\text { NegInf group } \\
7 \text { preterm } \\
\text { infants 9 suspected } \\
\text { infections }\end{array}$ \\
\hline Female & 6 & 4 \\
Gestational age (wk) & $27.8(25.8-27.9)$ & $28.0(26.4-29.1)$ \\
Birth weight $(\mathrm{g})$ & $879(797-1017)$ & $961(742-1275)$ \\
Days of antibiotic & $6(4.7-8.0)^{*}$ & $3(1.3-4.9)^{*}$ \\
$\quad$ therapy $(\mathrm{d})$ & $56(55-59)$ & $58(52-64)$ \\
Systolic BP $(\mathrm{mm} \mathrm{Hg})$ & $35(32-35)$ & $35(30-41)$ \\
Diastolic BP $(\mathrm{mm} \mathrm{Hg})$ & $43(40-44)$ & $41(38-47)$ \\
MAP (mm Hg) & $160(156-166)$ & $162(154-169)$ \\
Heart rate $(\mathrm{beats} / \mathrm{min})$ & $36.9(36.8-36.9)$ & $36.9(36.8-37.0)$ \\
Infant temperature $\left({ }^{\circ} \mathrm{C}\right)$ & $32.1(31.6-32.5)^{*}$ & $33.2(32.4-33.7)$ \\
Incubator temperature $\left({ }^{\circ} \mathrm{C}\right)$ & $35.6(35.4-35.7)$ & $35.6(35.3-35.9)$ \\
Measurement point & $30(28.8-33.4)$ & $28.5(26.7-34.6)$ \\
temperature $\left({ }^{\circ} \mathrm{C}\right)$ & $11.2(10.7-11.8)$ & $10.9(9.9-12.7)$ \\
Hematocrit $(\%)$ &
\end{tabular}

* PosInf group versus NegInf group: $p<0.05$.

suspected but not confirmed episodes in seven infants (NegInf group). No infant had both proven infection and suspected infection. Each episode was analyzed separately. Four infants remained well without any signs of infection during the entire study time. There were no significant differences in gestational week, birth weight (Table 1), Apgar scores, and duration of respiratory support (data not shown) between the two groups. In 16 of the 17 episodes of infection, IL-6 was increased [median (range): $36 \mathrm{pg} / \mathrm{mL}$ (17-9173)]. An increase in CRP was observed for five episodes [median (range): $1.3 \mathrm{mg} / \mathrm{dL}(0.7-12.5 \mathrm{mg} / \mathrm{dL})$ ]. Leukocyte count was also increased [median (range): 22.9 $\mathrm{g} / \mathrm{L}(7.7-71.5 \mathrm{~g} / \mathrm{L})]$. No blood culture was positive. Obviously, duration of antibiotic therapy was significant longer in PosInf group than in NegInf group (Table 1). Hb levels, body, and incubator temperatures did not change from day -5 to day -1 . Incubator temperature differed slightly but significantly between the PosInf and NegInf group (Table 1). The clinical score of the medical or the nursing staff varied widely and did not change significantly before infection or even on the day of infection.

Microcirculatory parameters. FSVD varied widely between individuals. Nevertheless, the paired comparison between day -5 and day -1 revealed a significant decrease of FSVD in the PosInf group (Table 2 and Fig. 1). In contrast, FSVD did not change significantly in the NegInf from day -5 to day -1 . In both groups, we did not find a

Table 2. Microcirculatory parameters on days -5 and -1 for the PosInf and NegInf groups as median (95\% CI)

\begin{tabular}{lccccc}
\hline & \multicolumn{3}{c}{ PosInf } & & \multicolumn{2}{c}{ NegInf } \\
\cline { 2 - 3 } \cline { 5 - 6 } & Day -5 & Day -1 & & Day -5 & Day -1 \\
\hline $\mathrm{n}$ & 12 & 16 & & 4 & 8 \\
FSVD & $234(189-257)$ & $231(200-257)$ & $208(128-283)$ & $209(175-244)$ \\
RBC & $312(290-360)$ & $298(260-350)$ & $443(301-567)$ & $353(274-473)$ \\
$\quad$ Vel & & & & & $16(13-17)$ \\
Diameter & $13.2(12-16)$ & $14.4(13-15)$ & & $19(15-22)$ & 16 \\
\hline
\end{tabular}



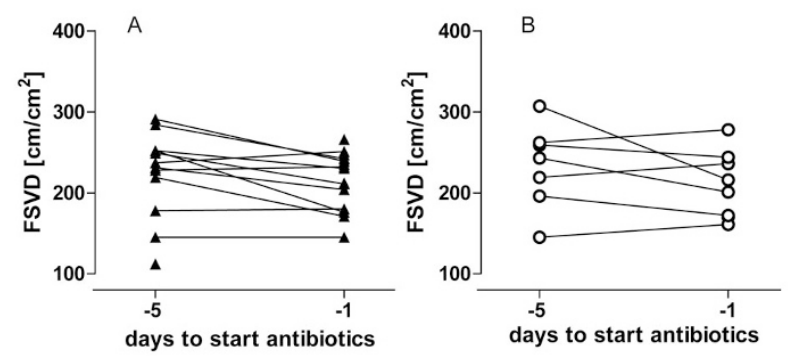

Figure 1. FSVD on day -5 and day -1 for the PosInf group $(A)$ and NegInf group $(B)$. In PosInf group, the FSVD decreased in seven pairs (mean decrease, $17 \%$; range, $8-30 \%$ ), increased in two pairs ( 2 and $6 \%$ ), and data for one of the two dates was missing in five episodes. In NegInf group, the FSVD decreased in four pairs (mean decrease, 17\%; range, 6-28\%), increased in four pairs (mean, 9\%; range, $6-12 \%$ ), data for one of the two dates was missing in one episode, and for one infant, data were only available from day 0 on.

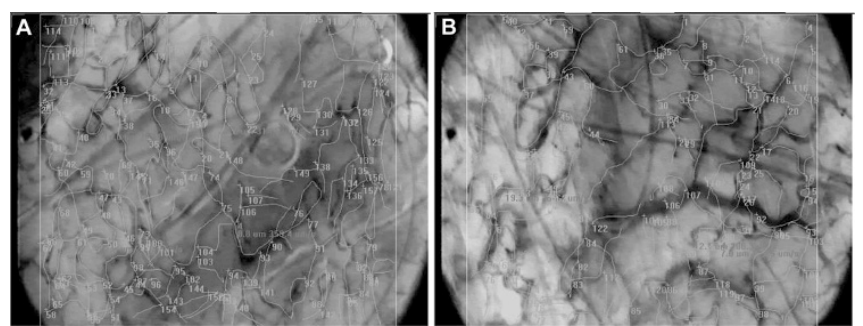

Figure 2. A, OPS scan of a premature infant (gestational age, $25+1 \mathrm{wk}$; birth weight, 570 g) $5 \mathrm{~d}$ before infection (day -5 of infection FSVD 291 $\mathrm{cm} / \mathrm{cm}^{2}$ ); $B$, OPS scan $1 \mathrm{~d}$ before the start of antibiotics (day -1 of infection: $239 \mathrm{~cm} / \mathrm{cm}^{2}$ ). The RBC perfused capillaries are traced in green color to determine the functional vessel density. Vessels with diameter from 6 to $<24$ $\mu \mathrm{m}$ are traced. In red marked vessels, RBC Vel was measured.

significant change from day 0 to day +5 . Figure 2 illustrates the decrease of FSVD of a premature infant with proven infection. There were no statistical significant changes in RBC Vel or vessel diameter from day -5 to day -1 in the interindividual as well as in the intraindividual comparison in the two groups (Table 2). When subdividing the PosInf group in before (day -5 until day -1 ) and while infection (day 0 until day +5 ), RBC Vel seemed to be reduced during the infection, but this change was not statistical significant (mean day -5 to day -1 : 306 (297-

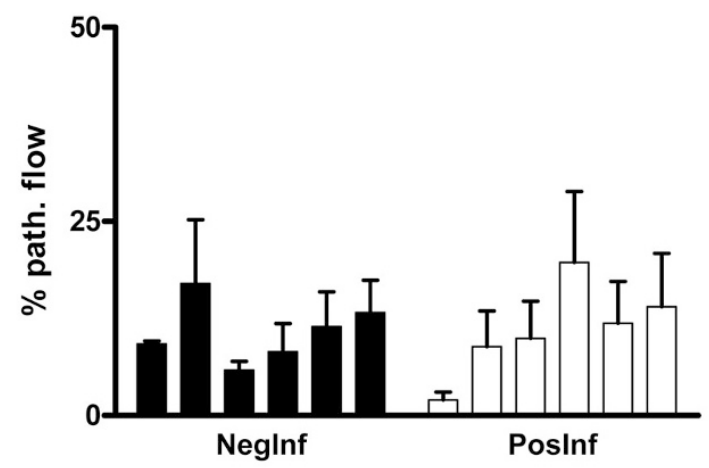

Figure 3. Fraction of capillaries with disturbed flow are shown as mean and SD for the two groups, PosInf and NegInf from day -5 until day 0 . There is no statistical difference between the groups or within the groups from day -5 to -1 .
334) $\mu \mathrm{m} / \mathrm{s}$ versus mean day 0 to day $+5: 280(283-317)$ $\mu \mathrm{m} / \mathrm{s} ; p=0.2)$. There was no significant correlation between RBC Vel and cardiovascular parameters for any group. Pathologic flow is rarely observed in preterm infants OPS imaging. We did not find a significant difference in percentage of disturbed flow between PosInf and NegInf. It seems that $\%$ pathologic flow rose slightly until day -2 in the PosInf, but this was not statistically significant (Fig. 3).

\section{DISCUSSION}

Our study demonstrates that changes in microcirculation play a crucial role in the development of septic organ dysfunction, but so far mainly macrocirculatory parameters, such as blood pressure, cardiac output and whole body oxygen delivery are measured in sepsis. Capillary refill time is an easy but quite subjective measurement with an endpoint that is difficult to define and is influenced significantly by environmental factors (16). FSVD is well validated and seems to be one of the best quantitative indicators of microvascular perfusion. As only RBC-perfused microvessels are included in its measurement FSVD can be seen as an indirect measure for oxygen transport (17). Despite the high inter- and intraindividual variability in our microcirculatory parameters, a change in FSVD associated with infection could be demonstrated for the first time. In infants with proven infection, we found a statistical significant decrease in FSVD from $5 \mathrm{~d}$ before infection to $1 \mathrm{~d}$ before clinical signs of infection and start of antibiotic treatment, whereas no such change could be demonstrated in infants with suspected but unproven infection. However, only intraindividual comparisons revealed the decrease in FSVD and not absolute values of FSVD.

This reduction of vessel density is in accordance to former animal experiments and human studies. De Baker $e t$ al. demonstrated a significantly diminished perfusion of small sublingual capillaries in adult patients with septic shock, which increased over time in survivors in comparison with nonsurvivors (15). We did not observe any changes during the course of infection in our premature infants, but none of our infants were in shock and most had only mild infection. Extremely low-birth weight infants are at high risk for secondary infection with significant mortality and long-term morbidity. They receive antibiotics at the first possible signs of infection to prevent deterioration. This may explain some of our findings, because we obtained our measurements very early in the course of infection. We were unable to demonstrate changes in the magnitude seen in adults with sepsis. Probably because of the very early treatment with antibiotics, none of our infants reached the severity of disease reported in the adult sepsis studies and none died. Nosocomial infections in preterm infants are frequently caused by Gram-positive Staphylococcus species and are subtle in onset. Dramatic changes in microcirculation cannot be expected. Mean FSVD decrease was $10 \%$ in our infants with proven infection. 
In RBC Vel, we found no change between day -5 and day -1 , this parameter merely showed a reduction while infection but without reaching statistical significance. Nevertheless, this tendency agrees with findings of other authors where blood flow decreases during infection or sepsis (18).

The mean vessel diameter was $14-19 \mu \mathrm{m}$ and was not different between the groups. This is not surprising because we evaluated only vessels with diameters $<24 \mu \mathrm{m}$ and were not able to obtain all vessel diameters for each image. At a hematocrit $<25 \%$, a systematic error of $4-5 \mu \mathrm{m}$ appears when determining the diameter by OPS imaging compared with intravital microscopy (8). Therefore, changes in diameter might be underestimated. This is not very likely in our study because all infants had hematocrit values above the critical level of $25 \%$. The new SDF imaging software might have an advantage in regard to this question, because vessel diameters are calculated automatically.

Incubator temperature was slightly but significantly lower in the PosInf group. This could be due to a higher body temperature in the infants with infection, which was counter regulated by the nurses by lowering the incubator temperature. On the other hand, incubator temperature did not differ between day -5 and day -1 . Therefore, the change in microcirculation is unlikely to be caused by lower incubator temperature. In the past, we have shown, that under the condition of "comfort temperature" (a minimal temperature difference between the abdominal wall and the extremities smaller than $2^{\circ} \mathrm{C}$ ) preterm infants with signs of a compromised microcirculation have higher skin blood flow than under "neutral temperature" (body surface temperature at the liver of $\sim 37^{\circ} \mathrm{C}$ regardless of temperature of the extremities) (19). There are major differences between the studies, which make comparisons very difficult. First, the method used to assess microcirculation, because the major part of the photoplethysmographic signal represents blood flow through arteriovenous shunts from a greater depth of the skin and larger vessels than in OPS. Second, incubator temperatures and body temperatures at "comfort temperature" are significantly higher than the temperatures in the current study.

Are the observed changes in microcirculatory parameters associated with infection? Although the mean arterial pressure of sick infants (PosInf group) was significantly lower than mean arterial blood pressure of healthy infants, it is unlikely that this factor is responsible for the microcirculatory alterations on its own. Animal experiments showed that changes in microcirculation are independent from arterial pressure. We also did not find a correlation between microvascular parameters and the blood pressure values of our preterm infants.

In adults, the microvascular alterations were demonstrated in the sublingual tissue. Because this vascular bed is not readily accessible in the newborn, since it would require a smaller probe design than currently available, we chose the transdermal application of OPS imaging. In premature neonates, microvascular malperfusion becomes more evident than in any other patient group. Their cardiovascular response to hypoperfusion is very restricted be- cause they are limited in heart rate response and their ability to increase myocardial contractility. As this is compensated by a profound vasoconstriction mainly of skin arteriovenous shunts the skin of neonates is somewhat a "shock organ," where distinct vasoconstriction indicates insufficient tissue perfusion (20). Thus, changes in the skin's microcirculation of a preterm newborn associated with infection can be expected. The significant decrease in FSVD $1 \mathrm{~d}$ before clinical or biochemical diagnosis of infection only became evident in PosInf group. On the basis of these results, the early detection of true infections could be supported by OPS imaging and consequently lead to a reduction of blood sampling and antibiotic therapy.

One problem of OPS imaging for microvascular monitoring in the human is the variability of vessels measured. When using the technique in the hamster skin fold chamber, it is possible to examine identical vessels over time as is in human capillaroscopy of the nail fold. But when using OPS imaging in humans, the vessels cannot be marked, and so it is not possible to measure identical vessels at different time points (9).

Another limiting factor is the application pressure, which cannot be controlled objectively without any special construction and may lead to pressure artifacts. The existing appliances, e.g., working with vacuum are hardly practicable in premature infants as the skin is too sensible and space in incubator is very restricted (21). If the probe is placed at the skin with uncontrolled high pressure, microvessels are compressed, and the blood flow is inhibited or even stagnates/ stops. This can lead to underestimated FSVD or incorrect measurements of RBC Vel. But with some experience it is possible to identify high application pressure, and so artifacts can be avoided (22).

At the time of the study neither the newer Sidestream Dark Field imaging probe, which produces better capillary quality than OPS, nor the faster automated analysis were available. It is speculative but quite possible that with better technology early changes in microcirculation as noninvasive markers of infection can be used as screening method.

In conclusion, we demonstrated that in premature infants microcirculatory alteration during infection could be measured by OPS imaging very early in the course of the disease. A decreasing FSVD correctly identified episodes of infections already $1 \mathrm{~d}$ before clinical suspicion led to treatment. Therefore, microcirculatory imaging might support early diagnosis of infections in premature infants and hence influence the clinical course of infection. However, these changes in FSVD during infection are not represented by absolute values but must be identified by daily intraindividual comparisons.

\section{REFERENCES}

1. Stoll BJ, Hansen N, Fanaroff AA, Wright LL, Carlo WA, Ehrenkranz RA, Lemons JA, Donovan EF, Stark AR, Tyson JE, Oh W, Bauer CR, Korones SB, Shankaran S, Laptook AR, Stevenson DK, Papile LA, Poole WK 2002 Late-onset sepsis in very low birth weight neonates: the experience of the NICHD Neonatal Research Network. Pediatrics 110:285-291

2. Franz AR, Bauer K, Schalk A, Garland SM, Bowman ED, Rex K, Nyholm C, Norman M, Bougatef A, Kron M, Mihatsch WA, Pohlandt F; International IL-8 Study Group 2004 Measurement of interleukin 8 in combination with C-reactive 
protein reduced unnecessary antibiotic therapy in newborn infants: a multicenter, randomized, controlled trial. Pediatrics 114:1-8

3. Laborada G, Rego M, Jain A, Guliano M, Stavola J, Ballabh P, Krauss AN, Auld PA, Nesin M 2003 Diagnostic value of cytokines and C-reactive protein in the first 24 hours of neonatal sepsis. Am J Perinatol 20:491-501

4. Farquhar I, Martin CM, Lam C, Potter R, Ellis CG, Sibbald WJ 1996 Decreased capillary density in vivo in bowel mucosa of rats with normotensive sepsis. J Surg Res 61:190-196

5. Piper RD, Pitt-Hyde M, Li F, Sibbald WJ, Potter RF 1996 Microcirculatory changes in rat skeletal muscle in sepsis. Am J Respir Crit Care Med 154:931-937

6. Sakr Y, Dubois MJ, De Backer D, Creteur J, Vincent JL 2004 Persistent microcirculatory alterations are associated with organ failure and death in patients with septic shock. Crit Care Med 32:1825-1831

7. Groner W, Winkelman JW, Harris AG, Ince C, Bouma GJ, Messmer K, Nadeau RG 1999 Orthogonal polarization spectral imaging: a new method for study of the microcirculation. Nat Med 5:1209-1212

8. Harris AG, Sinitsina I, Messmer K 2002 Validation of OPS imaging for microvascular measurements during isovolumic hemodilution and low hematocrits. Am J Physiol Heart Circ Physiol 282:H1502-H1509

9. Genzel-Boroviczény O, Stroetgen J, Harris AG, Messmer K, Christ F 2002 Orthogonal polarization spectral imaging (OPS): a novel method to measure the microcirculation in term and preterm infants transcutaneously. Pediatr Res 51:386-391

10. Kroth J, Weidlich K, Hiedl S, Nussbaum C, Christ F, Genzel-Boroviczeny O 2008 Functional vessel density in the first month of life in preterm neonates. Pediatr Res 64:567-571

11. Kuster H, Weiss M, Willeitner AE, Detlefsen S, Jeremias I, Zbojan J, Geiger R, Lipowsky G, Simbruner G 1998 Interleukin-1 receptor antagonist and interleukin-6 for early diagnosis of neonatal sepsis 2 days before clinical manifestation. Lancet $352: 1271-1277$
12. Thomas L 2007 C-Reaktives protein, in labor und diagnose. In: Thomas L (ed) Labor und Diagnose. TH-Books, Frankfurt, Germany, pp 1010-1022

13. Boerma EC, Mathura KR, van der Voort PH, Spronk PE, Ince C 2005 Quantifying bedside-derived imaging of microcirculatory abnormalities in septic patients: a prospective validation study. Crit Care 9:R601-R606

14. De Backer D, Hollenberg S, Boerma C, Goedhart P, Buchele G, Ospina-Tascon G, Dobbe I, Ince C 2007 How to evaluate the microcirculation: report of a round table conference. Crit Care 11:R101

15. De Backer D, Creteur J, Preiser JC, Dubois MJ, Vincent JL 2002 Microvascular blood flow is altered in patients with sepsis. Am J Respir Crit Care Med 166:98-104

16. LeFlore JL, Engle WD 2005 Capillary refill time is an unreliable indicator of cardiovascular status in term neonates. Adv Neonatal Care 5:147-154

17. Nolte D, Zeintl H, Steinbauer M, Pickelmann S, Messmer K 1995 Functional capillary density: an indicator of tissue perfusion? Int J Microcirc Clin Exp 15:244-249

18. Weinberg JR, Boyle P, Thomas K, Murphy K, Tooke JE, Guz A 1991 Capillary blood cell velocity is reduced in fever without hypotension. Int J Microcirc Clin Exp 10:13-19

19. Genzel-Boroviczeny O, Seidl T, Rieger-Fackeldey E, Abicht J, Christ F 2007 Impaired microvascular perfusion improves with increased incubator temperature in preterm infants. Pediatr Res 61:239-242

20. Christ F, Genzel-Boroviczény O, Schaudig S, Niklas M, Schiessler C, Strötgen J, Eifert S, Reichenspurner H, Harris AG, Messmer K 2000 Monitoring of the microcirculation in cardiac surgery and neonates using orthogonal polarization spectral imaging. In: Messmer K (ed) Progress in Applied Microcirculation. Karger, Basel, pp 82-93

21. Lindert J, Werner J, Redlin M, Kuppe H, Habazettl H, Pries AR 2002 OPS imaging of human microcirculation: a short technical report. J Vasc Res 39:368 372

22. Bezemer R, Khalilzada M, Ince C 2008 Recent advancements in microcirculatory image acquisition and analysis. In: Vincent JL (ed) Yearbook of Intensive Care and Emergency Medicine. Springer, Heidelberg, Germany, pp 677-690 